Supporting information

\title{
DMSA-Functionalized Mesoporous Alumina with a High Capacity for Selective Isolation of Immunoglobulin $\mathrm{G}$
}

Xi-Ming Wang, Peng-Fei Guo, Zheng-Jie Hu, Ming-Li Chen* and Jian-Hua Wang*

Research Center for Analytical Sciences, Department of Chemistry, College of

Sciences, Northeastern University, Box 332, Shenyang 110819, China

*Corresponding author.

*E-mail address: chenml@ mail.neu.edu.cn. (M.L. Chen), jianhuajrz@ mail.neu.edu.cn (J.H. Wang).

Tel: +86 24 83688944; Fax: +862483676698 


\section{Experimental section}

Chemicals and Reagents. Cetane trimethyl ammonium bromide (CTAB), aluminum acetylacetonate $\left(\mathrm{Al}(\mathrm{acac})_{3}\right)$, meso-2,3-dimercaptosuccinic acid (DMSA), tetrahydrofuran (THF), dimethylsulfoxide (DMSO), Coomassie brilliant blue G-250 and $\mathrm{R}-250$, ammonium hydroxide $\left(\mathrm{NH}_{4} \mathrm{OH}\right)$, sodium hydroxide, nitric acid, acetone, methanol and acetic acid and sodium chloride are purchased from Sinopharm Chemical Reagent Co. Ltd. (Shanghai, China). PEO $_{114}-b-P_{180}$ is purchased from Xirui Chemical Reagent Factory (Xi'an, China). The reagents are at least of analytical reagent grade and are used without further treatment or purification. Immunoglobulin G from human serum (IgG, I4506, 95\%, pI 8.0, Mr 150.0 kDa), conalbumin (ConA, C7786, 98\%, pI 6.8, Mr 77.7 kDa), transferrin (Trf, T3309, 98\%, pI 5.6, Mr 78.0 kDa), $\alpha$-lactalbumin from bovine milk ( $\alpha$-LA, M1882, 90\%, pI 4.2-4.5, Mr 16.0 kDa), lysozyme (Lys, L2879, pI 11.0) and bovine serum albumin (BSA, A3311, 98\%, pI 4.9, Mr 66.7 kDa) are purchased from Sigma-Aldrich (St. Louis, USA). The protein molecular weight marker (broad, 3452, Dalian, China, Takara Biotechnology Company) is a mixture of nine purified proteins ( $\mathrm{Mr}$ in kDa: myosin, 200; $\beta$-galactosidase, 116; phosphorylase B, 97.2; serum albumin, 66.4; ovalbumin, 44.3; carbonic anhydrase, 29; trypsin inhibitor, 20.1; lysozyme, 14.3; aprotinin, 6.5). Human serum samples are provided by healthy volunteer from the Hospital of Northeastern University, China.

Instrumentations. Scanning electron microscopic (SEM) images were collected on a LEO1430VP Scanning Electron Microscope (LEO, Germany). Transmission electron microscopy (TEM) images were taken on a JEM-1011 (JEOL, Japan) with an accelerating voltage of $100 \mathrm{kV} . \mathrm{N}_{2}$ adsorption/desorption experiments via the Autosorb-iQ2 instrument (Quantachrome, America). X-ray photoelectron 
spectroscopy (XPS) measurements were determined using a Thermo Scientific ESCALAB 250Xi electron spectrometer (Thermo Electron, America) with an Al Ka $1486.6 \mathrm{eV}$ excitation source. Fourier transform infrared spectra (FT-IR) were carried out on a Nicolet 6700 spectrophotometer (Thermo Electron, USA) from 4000 to 400 $\mathrm{cm}^{-1}$ with a resolution of $2.0 \mathrm{~cm}^{-1}$. Zeta potential measurements were carried out by a Nano ZS90 (Malvern, UK). The pH was adjusted by a PB-10 pH meter (Sartorius, Germany). The circular dichroism (CD) spectra are recorded with a MOS-450 spectrometer/polarimeter (Biologic Science Instrument, France) by using a $0.1 \mathrm{~cm}$ quartz cell.

The details concerning SDS-PAGE assay. For SDS-PAGE assay, all the sample aqueous solutions are mixed with loading buffer and boiling for $10 \mathrm{~min}$. Then the electrophoresis experiments are demonstrated on 5\% polyacrylamide stacking gel and $12 \%$ polyacrylamide separation gel at $90 \mathrm{~V}$ and $180 \mathrm{~V}$, respectively. The separation gel is stained with $0.1 \%(w / v)$ Coomassie Brilliant Blue R-250, and destained with a mixture solution containing $7.5 \%(\mathrm{v} / \mathrm{v})$ acetic acid and $5 \%(\mathrm{v} / \mathrm{v})$ methanol. 
Table S1. Elemental analysis of MA and DMSA-MA composites derived from XPS.

\begin{tabular}{ccccc}
\hline \multirow{2}{*}{ Materials } & \multicolumn{5}{c}{ Elements (\%) } \\
\cline { 2 - 5 } & $\mathrm{Al}$ & $\mathrm{O}$ & $\mathrm{C}$ & $\mathrm{S}$ \\
\hline MA & 34.1 & 52.49 & 13.41 & - \\
DMSA-MA & 30.18 & 51.15 & 16.26 & 2.41 \\
\hline
\end{tabular}



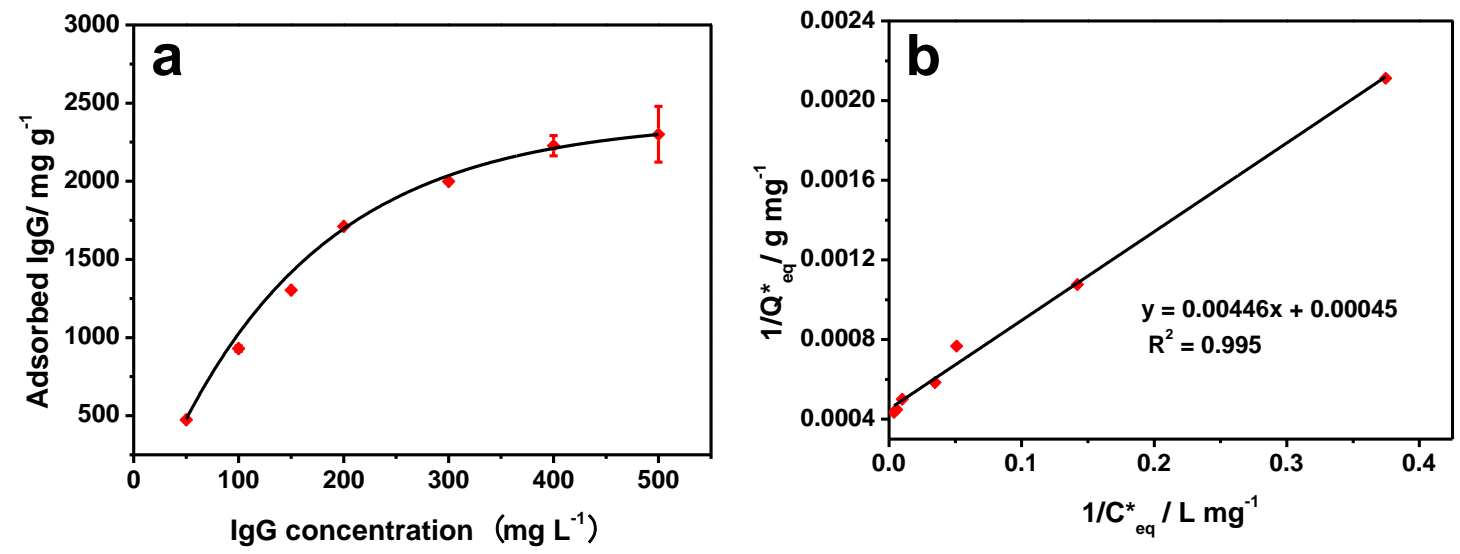

Figure S1. The adsorption isotherm of immunoglobulin G by DMSA-MA at pH 9 (a) and the plot of $1 / Q^{*}{ }_{\text {eq }}$ versus $1 / C^{*}{ }_{\text {eq }}(\mathrm{b})$. 